\title{
Putting the Diffuse Interstellar Bands on the Map - Literally!
}

\author{
J. Th. van Loon \\ Lennard-Jones Laboratories, Keele University, ST5 5BG, UK \\ email: j.t.van.loon@keele.ac.uk
}

\begin{abstract}
In a quest to further our understanding of the diffuse interstellar medium (ISM) as well as the unidentified carriers of the diffuse interstellar bands (DIBs), we are mapping DIBs across the sky using hundreds of hot stars as background torches - globular clusters (in particular $\omega$ Centauri), nearby stars in and around the Local Bubble, and stars within the Magellanic Clouds. I describe the results so far obtained and our current experiments.
\end{abstract}

Keywords. atlases, surveys, ISM: lines and bands, ISM: molecules, ISM: structure, globular clusters: individual: $\omega$ Centauri (NGC 5139), local interstellar matter, Magellanic Clouds

\section{Rationale}

While the carriers of the DIBs are not known, their correlations with better known constituents of the ISM suggest they are likely of molecular origin and reside in diffuse clouds and cloud surfaces (see the review by Daniel Welty, this volume). We can thus already start using DIBs to trace special ISM conditions, such as those related to the atomic-molecular transition or cloud irradiation effects, that otherwise may be rather elusive; hoping meanwhile to expose clues that help identify their carriers.

As no DIB has been seen in emission, we rely on background continuum sources to trace their absorption. Fields of stars, such as bright nearby stars, stars in open and globular clusters and stars in nearby resolved galaxies provide a means to construct sparse maps of the DIB absorption across a variety of ISM volumes. Compared to correlation diagrams, these maps add a spatial dimension, even rendering a 3-dimensional picture if stars at different known distances are observed.

\section{Experimental characteristics}

I here describe several of our recent and current DIB mapping experiments, summarised in Table 1. It all started with a spectroscopic survey of the post-main sequence stars in the "nearby" ( $\sim 5 \mathrm{kpc})$ most massive Galactic globular cluster, $\omega$ Centauri. This programme employed the powerful $2 \mathrm{dF}$ multi-object spectrograph on the 4-m AngloAustralian Telescope, which allows to place up to 400 fibres on positions on the sky within a huge, $2^{\circ}$-diameter field. The hot horizontal branch stars in that sample, further aided by their low metal content $\left(\sim 0.03 \mathrm{Z}_{\odot}\right)$ and high velocity $\left(\sim 230 \mathrm{~km} \mathrm{~s}^{-1}\right)$ with respect to the intervening ISM, turned out to be useful in mapping the Ca II K absorption. I here show (below) that in fact those blue spectra also display some DIBs. To do this more adequately, we designed a dedicated experiment with the enhanced $2 \mathrm{dF}$ facility $(\mathrm{AA} \Omega)$, allowing more spectral coverage at higher spectral resolution.

Likewise, we have just published DIB maps of the Tarantula Nebula, the famous "ministarburst" region in the Large Magellanic Cloud (at a distance of $50 \mathrm{kpc}$, and with $\sim 0.4$ $\mathrm{Z}_{\odot}$ and a recession velocity of $\sim 270 \mathrm{~km} \mathrm{~s}^{-1}$ ). This too was a "by-product" of a stellar 
Table 1. Overview of spectroscopic DIB mapping experiments.

\begin{tabular}{|c|c|c|c|c|c|c|}
\hline Target area & $N_{\star}$ & Facility & $\lambda / \Delta \lambda$ & $\mathrm{S} / \mathrm{N}$ & Main DIBs & Publications \\
\hline$\omega$ Centauri & $231 \in 1528$ & $\mathrm{AAT} / 2 \mathrm{dF}$ & 2,000 & $>50$ & 4428 & van Loon et al. (2007); here \\
\hline & 452 & $\mathrm{AAT} / \mathrm{AA} \Omega$ & 8,000 & 100 & 5780,5797 & van Loon et al. (2009); here \\
\hline Tarantula Nebula & 800 & VLT/FLAMES & 10,000 & 100 & 4428,6614 & van Loon et al. (2013) \\
\hline Magellanic Clouds & $>600$ & $\mathrm{AAT} / \mathrm{AA} \Omega$ & 8,000 & $>200$ & 5780,5797 & Bailey et al. (in prep.) \\
\hline Local Bubble South & 238 & $\mathrm{NTT} / \mathrm{EFOSC} 2$ & 8,000 & 1,000 & 5780,5797 & Bailey et al. (in prep.) \\
\hline North & 333 & INT/IDS & 2,000 & 1,000 & 5780,5797 & Farhang et al. (in prep.) \\
\hline
\end{tabular}

astrophysics programme - Evans et al. (2011). It utilised the FLAMES spectrograph, offering less multiplexity and coverage but a more sensitive 8-m Very Large Telescope in Chile. We have since conducted a dedicated survey of both the LMC and the Small Magellanic Cloud $\left(60 \mathrm{kpc}, \sim 0.2 \mathrm{Z}_{\odot}\right.$ and $\sim 160 \mathrm{~km} \mathrm{~s}^{-1}$ ) with AA $\Omega$; Keele University Ph.D. student Mandy Bailey has now finished the measurements from these spectra, and a publication of the results is expected to appear in 2014.

Inspired by the atomic-line 3-D mapping of the Local Bubble and its environs by Lallement et al. (2003) and Welsh et al. (2010) we have conducted an all-sky survey of DIB absorption towards 552 different early-type stars, mostly within a few hundred pc from the Sun. In contrast to the above efficient mapping experiments, this meant obtaining one spectrum at a time. Because interstellar absorption in such nearby stars is often inconspicuous, we aimed at a signal-to-noise ratio of 1000:1 or better; this incurs high demands on the calibration measurements as well. The Southern component of the survey was carried out at the 3.5-m New Technology Telescope in Chile, by Mandy Bailey, whilst the Northern component was added by Sharif University of Technology Ph.D. student Amin Farhang using Iranian time on the 2.5-m Isaac Newton Telescope in Spain. Publications arising from this programme are expected to appear in 2014.

\section{Extra-planar gas in front of $\omega$ Centauri}

The sightline towards $\omega$ Centauri is relatively simple, at $15^{\circ}$ Galactic latitude, with low reddening of $E(B-V) \sim 0.1 \mathrm{mag}$ (McDonald et al. 2009). Yet several components are seen in atomic absorption (Wood \& Bates 1994, and references therein): hot gas within the Local Bubble, neutral gas beyond it and including the Carina-Sagittarius spiral arm, and warm extra-planar gas at greater distances $\sim 0.5-1 \mathrm{kpc}$ above the Galactic Plane.

Judging from the marginally-resolved kinematics of the Ca II K line in our spectra, and various correlations, we concluded that the $5780 \AA$ DIB absorption towards $\omega$ Centauri originates mostly in the extra-planar gas. The high Ca II/Na I and $5780 \AA / 5797 \AA \mathrm{DIB}$ ratios in those sightlines indicate a $\sigma$-type environment, implying a strong radiation field or perhaps structures embedded within a hotter gas. However, some more neutral $\zeta$ type clouds were seen too, displaying stronger $5797 \AA$ DIB absorption; these are likely associated with the intervening spiral arm. Intriguingly, the DIBs were found to trace small-scale structure down to parsec scales (or below), both in the warm extra-planar gas and neutral gas of the Disc.

A re-analysis of our spectra reveals some more DIBs (Figure 1): at 4428, 4500 and 4762 $\AA$ in the hot-star spectra of van Loon et al. (2007), and near 5850, 6010 and $6200 \AA$ in those of van Loon et al. (2009). With more targets available as well, there is plenty of scope for more detailed studies in this interesting direction. 

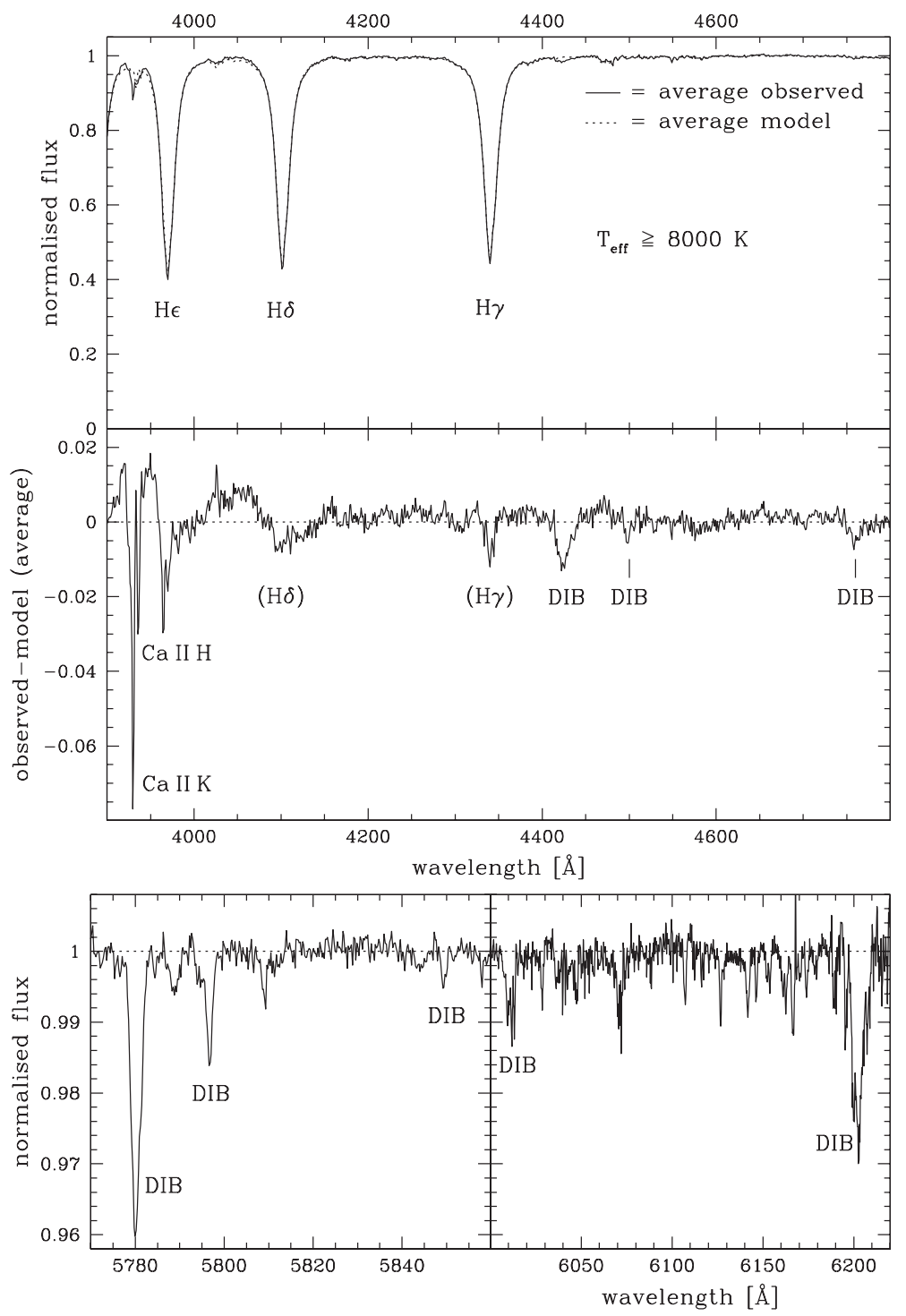

Figure 1. DIBs detected in the spectra of hot stars in $\omega$ Centauri. The top panel is based on the survey of van Loon et al. (2007) - but we identify the DIBs for the first time here; the bottom panel is based on van Loon et al. (2009) - who only presented the 5780 and $5797 \AA$ DIBs.

\section{The Magellanic Clouds and their Galactic foreground}

Our Magellanic DIB mapping exercise has thus far concentrated on the Tarantula Nebula. This is a complex region characterised by multi-phase ISM including molecular clouds (e.g., as traced in absorption by Tatton et al. 2013) and bubbles filled with hot gas, subject to a strong radiation field from the many massive early-type stars in the central cluster R 136 and other young associations (cf. Evans et al. 2011).

We were able to separate the Magellanic absorption from that arising in the Galactic foreground. The latter resembled the extra-planar environment seen in the $\omega$ Centauri maps. Small-scale structure within the LMC was probed both spatially (down to $\sim 10 \mathrm{pc}$ ) and kinematically ( $\sim 10 \mathrm{~km} \mathrm{~s}^{-1}$; up to six components in Na I D). While the $5780 \AA / 5797 \AA$ 


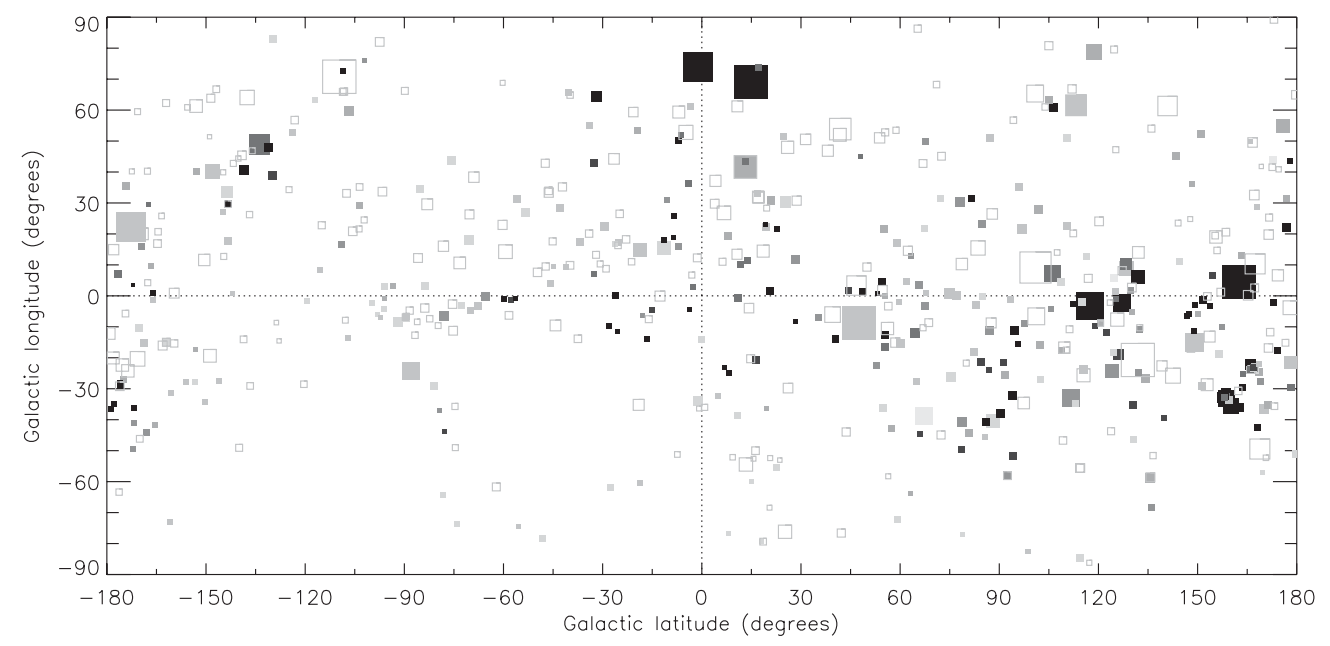

Figure 2. Preliminary Galactic map of the Local Bubble measurements of the $5780 \AA$ DIB, combining the Southern (Bailey et al.) and Northern (Farhang et al.) surveys. Darker symbols have stronger absorption, and larger symbols are nearer; open symbols are non-detections.

DIB ratio in the Tarantula Nebula is generally high, regional variations occur in the DIBs indicating particularly harsh environments towards the North and West (in the direction of super-bubbles) and in the immediate vicinity of OB-type associations, with more shielded conditions towards the South (where the molecular ridge starts). The $4428 \AA$ DIB proved relatively strong in and around the Tarantula Nebula, whilst the $5780 \AA$ DIB could be more strongly confined to cloud skins.

Even if accounting for the reduced metal content of the LMC, the 5780, 5797 and $6614 \AA$ DIBs are weak. Welty et al. (2006) and Cox et al. (2006, 2007) attributed this to the strong radiation field. We proposed to also consider the nitrogen depletion in the Magellanic Clouds (cf. van Loon et al. 2010a,b; see also Veronica Bierbaum's contribution to these proceedings). I would also suggest to examine any relation with the depletion of Poly-cyclic Aromatic Hydrocarbons (cf. Sandstrom et al. 2012).

The relatively little studied set of DIBs at 4727,4762 and $4780 \AA$ may prove quite interesting, as we found some tentative evidence for differences among these features between the Tarantula Nebula and the Galactic foreground.

While the results of our galaxy-wide Magellanic Cloud maps will be presented elsewhere, we can already reveal that in the SMC very few sightlines show DIBs. However, when it does it sometimes is fairly strong. We must consider that, possibly, the conditions under which DIBs are seen might occur in slightly different parts of clouds that have a reduced metal content (see also the behaviour of interstellar ice - Oliveira et al. 2013).

Our Magellanic Clouds surveys also offer a detailed view of the high Galactic latitude DIB absorption, adding to our all-sky survey of nearby stars to probe structure on scales of $0.1 \mathrm{pc}$ in and around the Local Bubble.

\section{The Local Bubble}

We have managed to obtain reasonable all-sky coverage, maintaining also a range in distance so we can probe the 3-D structure of the Local Bubble and its surrounding neutral medium (Figure 2; Bailey et al. and Farhang et al., in preparation; see also Amin Farhang's contribution to these proceedings). 
While there is some concentration of $5780 \AA$ DIB absorption towards the Galactic Plane for stars outside the Local Bubble (beyond $~ 100-200 \mathrm{pc}$ ), there also appear to be some DIB structures very nearby $(\ll 100 \mathrm{pc})$, well inside the Local Bubble and including some high-latitude clouds.

No less interesting are the weak or null detections, which are not restricted to nearby or high-latitude stars. It is not uncommon for the $5780 \AA$ DIB to be stronger in a nearby star seen in a direction close to that of a more distant star with weaker DIB absorption. Indeed, there is a generally poor correlation between DIB equivalent width and distance. The Milky Way as seen in the $5780 \AA$ DIB appears rather patchy and porous.

\section{Acknowledgements}

I am grateful to the Universe for being so wonderfully mysterious.

\section{References}

Cox, N. L. J., Cordiner, M. A., Cami, J., Foing, B. H., Sarre, P. J., Kaper, L., \& Ehrenfreund, P. 2006, $A \mathscr{E} A, 447,991$

Cox, N. L. J., et al. 2007, A\&BA, 470, 941

Evans, C. J., et al. 2011, A\&A, 530, A108

Lallement, R., Welsh, B. Y., Vergely, J.-L., Crifo, F., \& Sfeir, D. 2003, A\&\&A, 411, 447

McDonald, I., van Loon, J. T. h., Decin, L., Boyer, M. L., Dupree, A. K., Evans, A., Gehrz, R. D., \& Woodward, C. E. 2009, MNRAS, 394, 831

Oliveira, J. M., et al. 2013, MNRAS, 428, 3001

Sandstrom, K. M., et al. 2012, ApJ, 744, 20

Tatton, B. L., et al. 2013, A\&A, 554, A33

van Loon, J. T. h., van Leeuwen, F., Smalley, B. S., Smith, A. W., Lyons, N. A., McDonald, I., \& Boyer, M. L. 2007, MNRAS, 382, 1353

van Loon, J. T. h., Smith, K. T., McDonald, I., Sarre, P. J., Fossey, S. J., \& Sharp, R. G. 2009, MNRAS, 399, 195

van Loon, J. T. h., et al. 2010a, AJ, 139, 68

van Loon, J. T. h., Oliveira, J. M., Gordon, K. D., Sloan, G. C., \& Engelbracht, C. W. 2010b, AJ, 139, 1553

van Loon, J. T. h., et al. 2013, A\&A, 550, A108

Welsh, B. Y., Lallement, R., Vergely, J.-L., \& Raimond, S. 2010, A\&A, 510, A54

Welty, D. E., Federman, S. R., Gredel, R., Thorburn, J. A., \& Lambert, D. L. 2006, ApJS, 165, 138

Wood, K. D. \& Bates, B. 1994, MNRAS, 267, 660 